\title{
Fatal Encephalitis in a Case of Hypereosinophilic Syndrome: MRI and Autopsy Findings
}

\author{
Zen Kobayashi ${ }^{1,2}$, Kuniaki Tsuchiya ${ }^{2}$, Hiroshi Komachi ${ }^{3}$, Kazunori Miki ${ }^{4}$, \\ Osamu Yokota ${ }^{5}$, Tetsuaki Arai ${ }^{2,6}$, Hirotomo Miake ${ }^{7}$, Hideki Ishizu ${ }^{8}$, \\ Haruhiko Akiyama $^{2}$ and Hidehiro Mizusawa ${ }^{1}$
}

\begin{abstract}
A 34-year-old man developed fever and headache, followed by finger tremor and gait disturbance, and was admitted to our hospital about two months after onset. Blood tests showed a white blood cell count of $32,600 / \mu \mathrm{L}$ with an eosinophil count of $22,300 / \mu \mathrm{L}$. There was no evidence of allergic drug reaction or parasitic infection. Cerebrospinal fluid examination demonstrated mononuclear pleocytosis without eosinophils or atypical cells. Brain MRI showed symmetric lesions bilaterally in the medial temporal lobe, frontobasal and insular regions and medulla oblongata. Herpes simplex virus-DNA was negative in the cerebrospinal fluid. The patient died about four months after onset. Histopathologically, there was infiltration of T cells, B cells and macrophages throughout the whole brain, but eosinophils or atypical cells were absent. Immunohistochemistry for herpes simplex virus type 1 and human herpesvirus 6 was negative. This case suggests that fatal encephalitis may develop in association with hypereosinophilic syndrome.
\end{abstract}

Key words: fatal encephalitis, eosinophil, hypereosinophilic syndrome, lymphocyte

(Intern Med 50: 1219-1225, 2011)

(DOI: 10.2169/internalmedicine.50.4570)

\section{Introduction}

Hypereosinophilic syndrome (HES) refers to a group of disorders originally characterized by persistent ( $>6$ months) and marked hypereosinophilia $(>1,500 / \mu \mathrm{L})$ not due to an underlying disease, and is accompanied by damage to at least one target organ or tissue $(1,2)$. However, HES patients with obvious tissue damage should not be observed for 6 months before diagnosis and subsequent treatment (2). In HES, nervous system involvement is sometimes seen and manifests as peripheral neuropathy, recurrent optic neuritis (3), transverse myelitis (4), subdural effusion (5), encephalopathy (6-8), eosinophilic meningitis $(9,10)$, benign encephalitis $(11,12)$, mass lesion $(10,13,14)$, brain infarc- tion $(6,15-19)$, hemorrhage $(6,19)$, and cerebral venous sinus thrombosis $(20,21)$. The pathogeneses of neurologic dysfunction in HES include [1] direct infiltration of eosinophils into neural tissue, [2] direct secretion of eosinophil products onto neurons or secretion of intracytoplasmic contents into circulation, with subsequent damage to neural tissue, and [3] embolic brain infarction (22). Here, we report a 34-year-old man with HES showing fatal encephalitis, which has not been reported in HES.

\section{Case Report}

The patient was a 34-year-old healthy and well-developed Japanese man. His past history did not include allergic rhinitis or bronchial asthma. In July, he presented with general

${ }^{1}$ Department of Neurology and Neurological Science, Graduate School, Tokyo Medical and Dental University, Japan, ${ }^{2}$ Department of Psychogeriatrics, Tokyo Institute of Psychiatry, Japan, ${ }^{3}$ University Health Center, Chuo University, Japan, ${ }^{4}$ Department of Neurology, Toride Kyodo General Hospital, Japan, ${ }^{5}$ Department of Neuropsychiatry, Okayama University Graduate School of Medicine, Dentistry and Pharmaceutical Sciences, Japan, ${ }^{6}$ Department of Psychiatry, Graduate School of Comprehensive Human Sciences, University of Tsukuba, Japan, ${ }^{7}$ Department of Neurology, National Disaster Medical Center, Japan and ${ }^{8}$ Department of Laboratory Medicine, Zikei Institute of Psychiatry, Japan Received for publication September 21, 2010; Accepted for publication February 6, 2011

Correspondence to Dr. Zen Kobayashi, zen@bg7.so-net.ne.jp 
fatigue and appetite loss. In August, these symptoms gradually worsened and fever, headache, finger tremor, and vertigo also appeared. He consulted a doctor, and was diagnosed as having a common cold. However, he became unable to write or walk in September, and was admitted to our hospital. On admission, his body temperature was $38.5^{\circ} \mathrm{C}$, and other vital signs were within normal limits. Consciousness was slightly disturbed; Japan Coma Scale was 1 and Glasgow Coma Scale was 14. Neurological examination demonstrated neck stiffness, Kernig's sign, intention tremor and limb and truncal ataxia. The cranial nerves were not involved and sensory disturbance was not apparent. There were no abnormalities in the skin or joint. Blood tests showed white blood cell count (WBC) of $32,600 / \mu \mathrm{L}$ comprising stab form cells $4.0 \%$, segmented cells $17.5 \%$, lymphocytes $6.5 \%$, and eosinophils $68.5 \%$ (absolute count of $22,300 / \mu \mathrm{L})$. Atypical cells were not demonstrated. Hemoglobin $(\mathrm{Hb})$ was $13.3 \mathrm{~g} / \mathrm{dL}$, platelet count (Plt) $29.9 \times 10^{4} / \mu \mathrm{L}$, albumin (Alb) $3.8 \mathrm{~g} / \mathrm{dL}$, urea nitrogen (UN) $7.9 \mathrm{mg} / \mathrm{dL}$, creatinine (Cre) $1.22 \mathrm{mg} / \mathrm{dL}$ (normal range<1.2), sodium 136 $\mathrm{mEq} / \mathrm{L}$, potassium $4.1 \mathrm{mEq} / \mathrm{L}$, aspartate aminotransferase (AST) $36 \mathrm{IU} / \mathrm{L}$, alanine aminotransferase (ALT) $32 \mathrm{IU} / \mathrm{L}$, lactate dehydrogenase (LDH) 518 IU/L (normal range: 100250), blood sugar (BS) $104 \mathrm{mg} / \mathrm{dL}$, C-reactive protein (CRP) $0.1 \mathrm{~g} / \mathrm{dL}$, and IgE $229 \mathrm{IU} / \mathrm{mL}$ (not elevated). Antinuclear antibodies, anti-DNA antibodies, myeloperoxidase (MPO) specific anti-neutrophil cytoplasmic antibodies (ANCA) and rheumatoid arthritis test were negative. Chest $\mathrm{X}$-ray demonstrated a cardio-thoracic ratio of $52 \%$ and no abnormalities in the bilateral lungs. There was no evidence of allergic drug reaction or parasitic infection. Cerebrospinal fluid (CSF) examination demonstrated mononuclear pleocytosis of $71 / \mathrm{mm}^{3}$, total protein $119.2 \mathrm{mg} / \mathrm{dL}$, glucose $40 \mathrm{mg} /$ $\mathrm{dL}$, chloride $118 \mathrm{mEq} / \mathrm{L}$. Eosinophils or atypical cells were absent. His consciousness gradually deteriorated after admission. Blood test seven days after admission showed a reduction of the absolute count of the eosinophils $(7,728 / \mu \mathrm{L})$, but brain MRI demonstrated lesions localized in the bilateral medial temporal lobe and medulla oblongata. Acyclovir was started with methylprednisolone pulse therapy (1 g/day, 3 days) on the same day. Herpes simplex virus (HSV)-DNA was later determined to be negative in the CSF. Electroencephalography 11 days after admission demonstrated that basic rhythm was 1-2 Hz delta waves with occasional $9 \mathrm{~Hz}$ $\alpha$ activities. Bone marrow aspiration demonstrated a nucleated cell count of $21.8 \times 10^{4} / \mu \mathrm{L}$ (within normal limits) with proliferation of eosinophilic cells $(48.2 \%)$, but atypical cells were absent. Fever and consciousness disturbance disappeared after methylprednisolone pulse therapy, and the eosinophilia also disappeared at 11 days after admission. CSF examination 18 days after admission demonstrated cell count of $24 / \mathrm{mm}^{3}$, total protein $106.1 \mathrm{mg} / \mathrm{dL}$, and LDH 20 IU/L. However, brain MRI 21 days after admission demonstrated extensive and symmetric lesions bilaterally in the medial temporal lobe, frontobasal and insular regions, genu and posterior limb of the internal capsule, and medulla ob- longata (Fig. 1). The left middle frontal gyrus and caudate nucleus were also involved (Fig. 1). Urinary retention became evident and was considered as neurogenic bladder because urological examination showed no abnormalities in the bladder, prostate, or testis. Subsequently, he developed headache and consciousness disturbance, and finally required a respirator 45 days after admission. Thereafter, generalized convulsion occurred and repeated for six days. The patient died of bronchopneumonia, disseminated intravascular coagulation, and multiple organ failure 69 days after admission. There was no evidence of the presence of malignant neoplasm throughout the clinical course. Because the cause of eosinophilia was unknown, we made a diagnosis of HES.

\section{Neuropathological findings}

Pathological analysis was limited to the brain. Brain weight was $1,535 \mathrm{~g}$ after fixation. Macroscopically, there was a limited amount of fresh subdural hemorrhage. The pia matter was slightly cloudy. Vessels on the brain surface were markedly congested. Swelling was evident throughout the whole brain including the brainstem and cerebellum. Mild uncal herniation was seen bilaterally. On sections, the cerebrum, brainstem and cerebellum were edematous, and the right hippocampus appeared gray. Pallor of the substantia nigra was seen. Fresh hemorrhage measuring approximately 2 $\mathrm{mm}$ in diameter was seen in the left pontine base. Spinal cord was not available for analysis.

Ten $\mu$ m-thick hemispheric sections were prepared at the plane of the right orbital gyrus, right caudate head, right globus pallidus, right mamillary body, right posterior hippocampus, and left posterior lobe. The sections of the midbrain, pons, medulla oblongata, and cerebellum were also prepared. These sections were stained with hematoxylineosin (H\&E) and Klüver-Barrera (KB). Immunohistochemistry for CD3 (T cell markers, SP3, rabbit, monoclonal, 1:100, Nichirei, Tokyo), CD20 (B cell marker, L26, mouse, monoclonal, 1:100, Dako, Glostrup), CD68 (macrophage/microglia marker, KP1, mouse, monoclonal, 1:100, Dako, Glostrup), collagen type IV (rabbit, 1:10,000, Polysciences, Warrington), HSV type 1 (rabbit, polyclonal, 1:100, Dako, Glostrup), and human herpesvirus 6 (HHV6, P101, mouse, monoclonal, 1:50, Millipore, Billerica) was also performed.

$\mathrm{KB}$ stain of the right cerebrum demonstrated severe loss of myelin in the medial and superior parts of the temporal lobe, insular region, and inferior part of the frontal lobe (Fig. 2). In these areas, cellular infiltration, glial proliferation and necrosis were prominent, and normal laminar structures of the cerebral cortex were not preserved (Fig. 3a, b). In the right hippocampus, pyramidal cells and dentate granular cells were completely lost (Fig. 3c, d). Perivascular and parenchymal cellular infiltration was seen not only in these areas but also throughout the gray and white matter of the whole brain including the brainstem and cerebellum. The leptomeninges (Fig. 3b) and ventricles were also involved. The lesions generally showed loss of neurons and/or myelin. Some of the remaining neurons showed atrophic and eosino- 

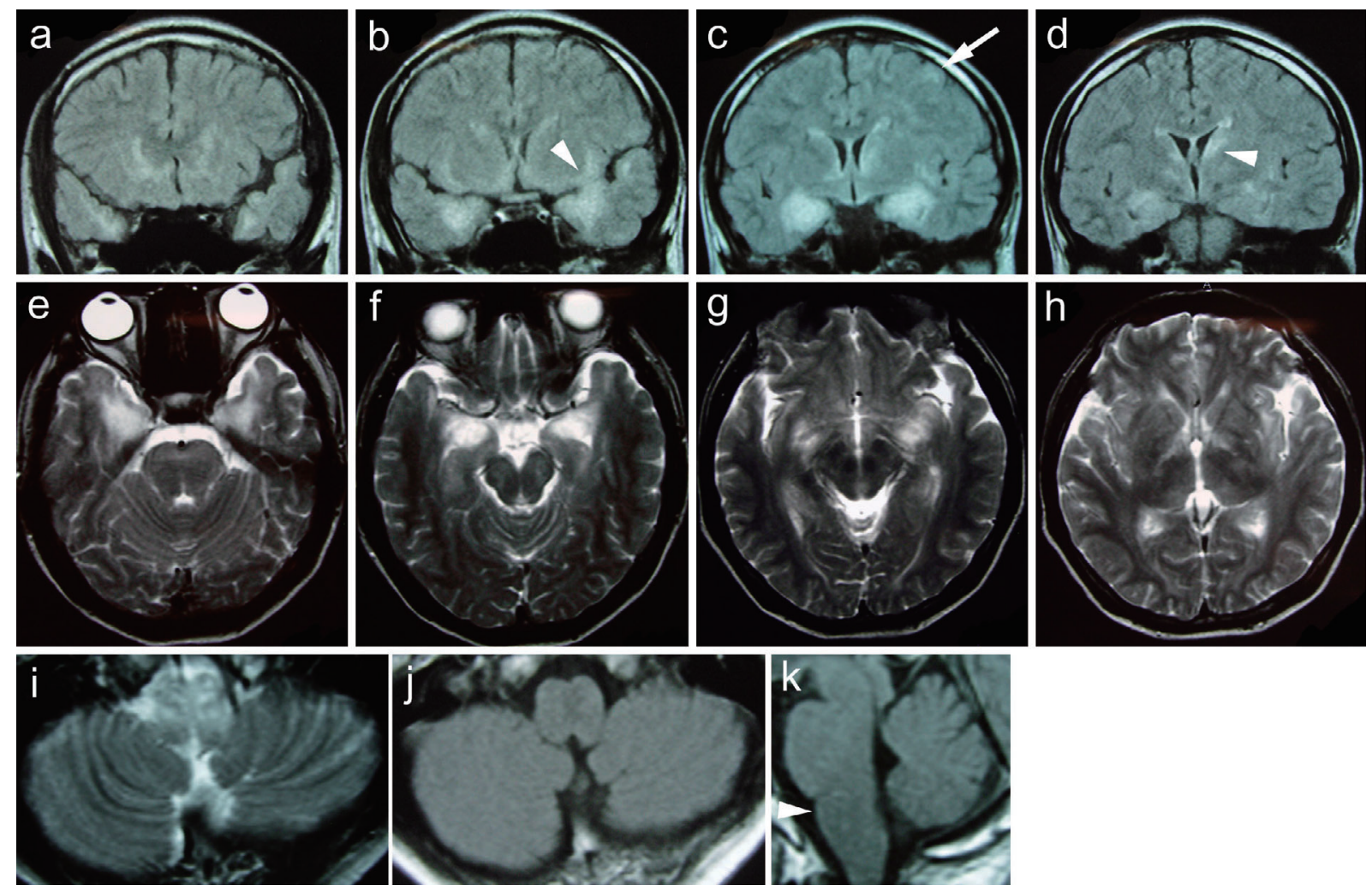

Figure 1. (a)-(d) Coronal image of fluid-attenuated inversion recovery (FLAIR) 21 days after admission demonstrated hyperintensities in the bilateral medial temporal lobe, frontobasal and insular regions, and left middle frontal gyrus (c, arrow) and caudate nucleus (d, arrowhead). The arrowhead in (b) indicates the left frontobasal and insular regions. (e)-(i) Axial image of T2-weighted MRI showed hyperintensities in the bilateral medial temporal lobe (e, f), insular region (g, h), genu and posterior limb of the internal capsule (h), and medulla oblongata (i). (j) Axial image of T1-weighted MRI demonstrated swelling and a focal low intensity area in the medulla oblongata. (k) Sagittal image of T1-weighted MRI also showed swelling of the medulla oblongata (arrowhead).
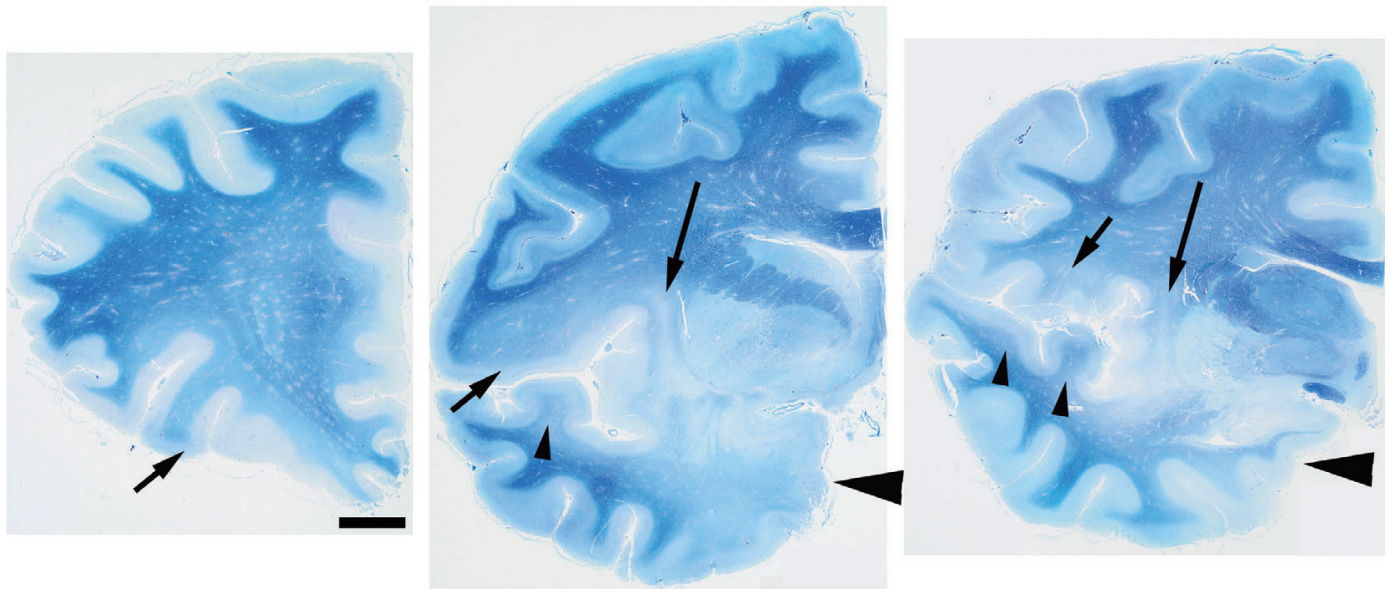

Figure 2. Klüver-Barrera (KB) stain of the right frontal and temporal lobe showed swelling of the brain, and severe loss of myelin in the medial (large arrowheads) and superior parts (small arrowheads) of the temporal lobe, insular region (long arrows), and inferior part of the frontal lobe (short arrows). Bar=1 cm.

philic changes while neuronophagia was not apparent. The infiltrating cells comprised lymphocytes and macrophages, and did not include eosinophils or atypical cells. These cells were easily found around medium-sized (50-250 $\mu \mathrm{m}$ in di- ameter) and small-sized (20-49 $\mu \mathrm{m}$ in diameter) arteries and veins (Fig. 3e, f), and generally showed multiple layers (Fig. 4). The vessel walls were relatively intact, indicating that vasculitis seemed unlikely. There was no vessel occlu- 


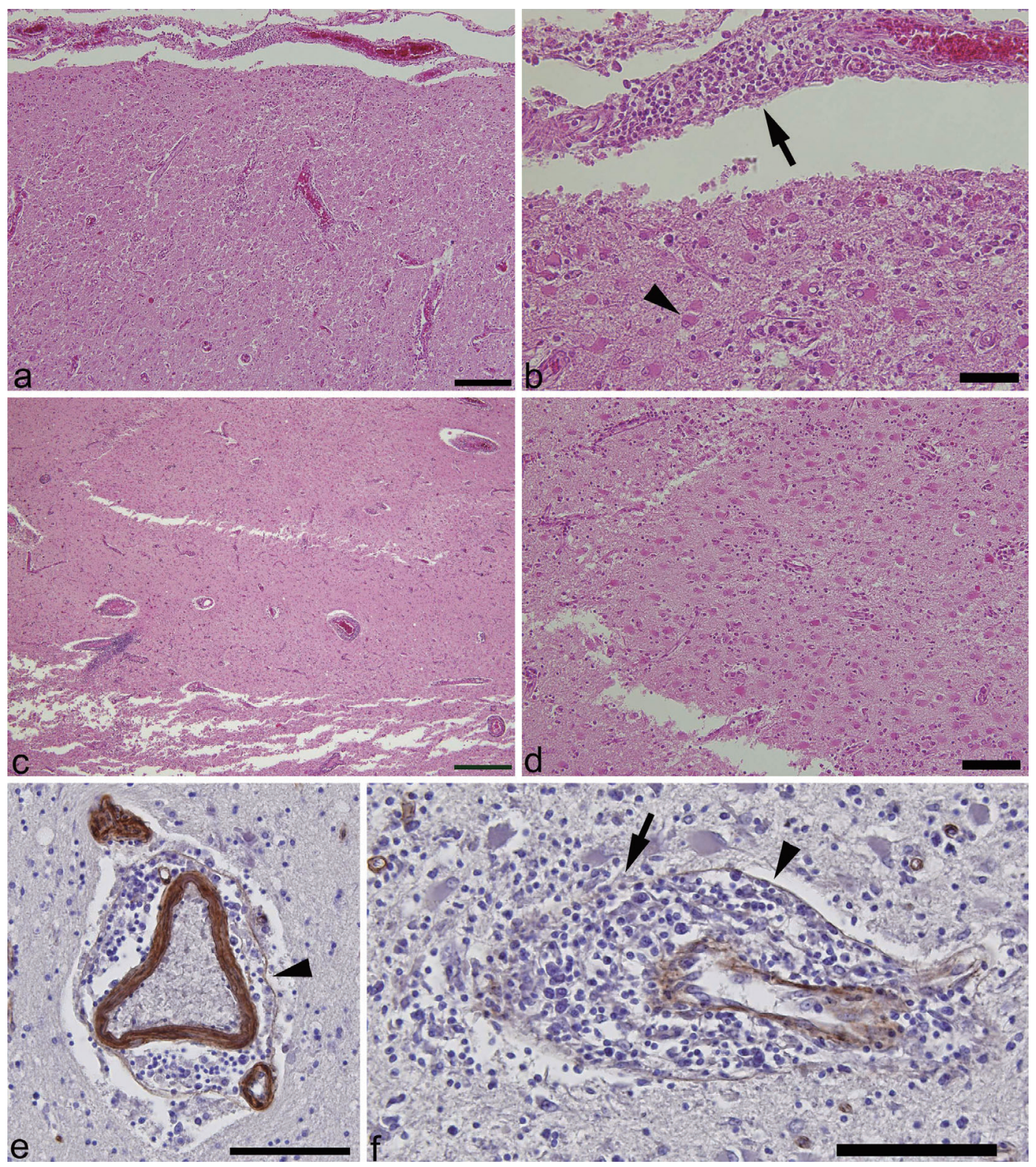

Figure 3. (a) The right lateral occipitotemporal cortex was severely affected, and normal laminar structures of the cortex were not preserved. (b) Lymphocytic infiltration (arrow) was seen in the pia matter, and gemistocytic astrocytes (arrowhead) were observed in the lateral occipitotemporal cortex. (c) Perivascular cellular infiltrations were demonstrated in the right hippocampus. Pyramidal cells of the subiculum and dentate granular cells were completely lost. (d) Cellular infiltration and gemistocytic astrocytes were seen in the CA4 and dentate gyrus. (e), (f) Infiltrating cells were found around both an artery (e) and a vein (f), and usually resided within the reticulin network (e), which was sometimes broken (f, arrow). The arrowheads in (e) and (f) indicate the outer boundaries of the reticulin network. (a)-(d) Hematoxylin and Eosin staining, (e), (f) collagen type IV and Hematoxylin (counterstain). Scale bars $=200 \mu \mathrm{m}$ (a), $50 \mu \mathrm{m}$ (b), $500 \mu \mathrm{m}$ (c), and $100 \mu \mathrm{m}$ (d-f).

sion. Pinpoint fresh hemorrhages were seen in the left pontine base and right midbrain. Cowdry type A inclusion was not found anywhere. Immunohistochemistry demonstrated that infiltrating cells of the parenchyma were CD3 positive cells and CD68 positive cells, whereas those of the perivascular areas included many CD20 positive cells (Fig. 4). Immunohistochemistry for HSV-1 and HHV-6 was negative.

Discussion

The clinicopathological characteristics of this case were as follows: 1) subacute course lasting for about two months between the onset and admission, 2) presence of HES, 3) almost symmetric MRI lesions compatible with limbic encephalitis, 4) infiltration of $\mathrm{T}$ and $\mathrm{B}$ cells, and macrophages without eosinophils or atypical cells throughout the whole 

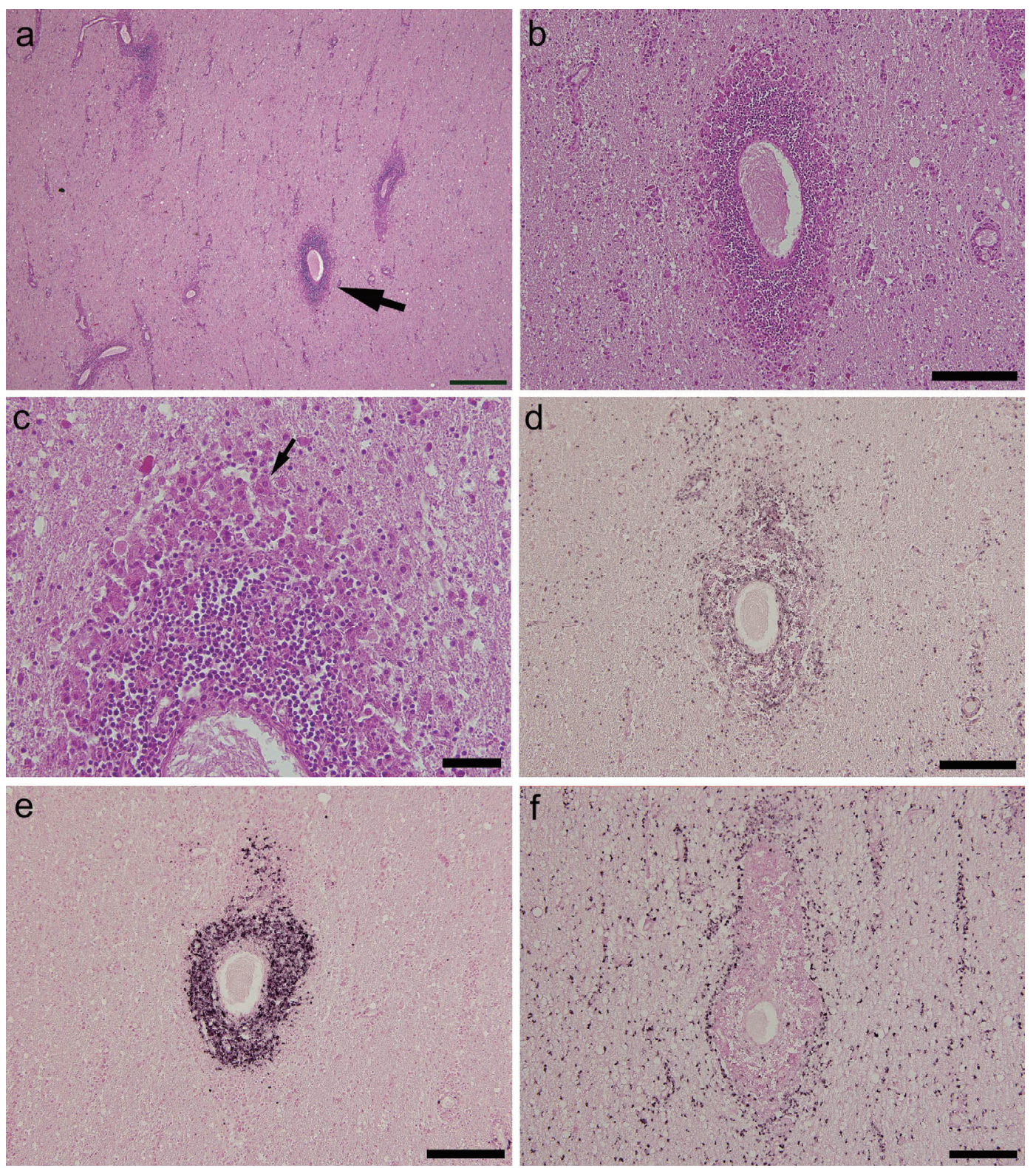

Figure 4. (a) Perivascular cellular infiltrations were seen in the white matter of the frontal lobe. The medium-sized vessel indicated with an arrow is shown in (b). (b), (d), (e), and (f) are serial sections. (c) Infiltrating cells composed of lymphocytes and macrophages showed multiple layers around the vessel wall. The arrow indicates a macrophage. (d) CD3-positive cells were mainly demonstrated in the perivascular area, but were also seen in the parenchyma. (e) Many CD20-positive cells were seen only in the perivascular area. (f) CD68-positive cells were mainly observed around the perivascular lymphocytes, but were also seen in the parenchyma. a-c Hematoxylin and Eosin staining. Scale bars $=500 \mu \mathrm{m}(\mathrm{a}), 200 \mu \mathrm{m}(\mathrm{b}, \mathrm{d}-\mathbf{f})$, and $50 \mu \mathrm{m}$ (c).

brain. The neuronal loss seen in the hippocampus and cerebral cortex may have been due to repeated convulsions. Although the reason for the deterioration of consciousness seen in the late stage of the disease was unclear, it may be non-convulsive status epilepticus because the convulsion appeared after the deterioration of consciousness.

While the cause of HES is largely unknown, two major subtypes, myeloproliferative and lymphocytic variants are well established $(1,2)$. The former is actually chronic eosinophilic leukemia with Fip1-like-platelet-derived growth factor receptor alpha chain (FIP1 L1-PDGFRA) mutation, and the latter is associated with T-cell clones producing interleukin-5 (IL-5) and can evolve into lymphoma (2). In the present case, myeloproliferative HES seems unlikely based on the findings on bone marrow aspiration. On the other hand, the predominant $\mathrm{B}$ cells in the perivascular areas demonstrated by autopsy were not consistent with lymphocytic HES caused by T-cell clones.

To date, cellular infiltration into the brain in HES has been verified in three cases $(10,13,14)$. Among these cases, infiltrating cells of the case reported by Battineni et al comprised not only "scattered eosinophils" but also lympho- 
cytes, histiocytes and plasma cells (14). In other words, eosinophils were not the main component of the infiltrating cells, as seen in the present case.

The differential diagnosis of the present case may include HSV type 1 encephalitis, HHV6 encephalitis, Churg-Straus syndrome, and autoimmune paraneoplastic or nonparaneoplastic encephalitis. First, HSV type 1 encephalitis seemed unlikely based on the absence of HSV-DNA in the $\mathrm{CSF}$, lack of hemorrhagic necrosis or Cowdry type A inclusion on histological examination, and negative HSV type 1 on immunohistochemistry. Second, the combination of hypereosinophilia and encephalitis seen in our case suggests HHV6 encephalitis, which sometimes occurs as druginduced hypersensitivity syndrome (23-27). This syndrome is related to reactivation of HHV6 caused by phenytoin, phenobarbital, allopurinol or sulfadiazine, and can manifest as benign limbic encephalitis. We excluded this encephalitis because the patient had not taken any of these drugs before the onset of disease, and presented an unfavorable course with negative HHV-6 on immunohistochemistry. Third, Churg-Straus syndrome can be ruled out because allergic rhinitis, bronchial asthma, or MPO-ANCA was absent clinically, and infiltration of eosinophils was not demonstrated histopathologically. Finally, it may be difficult to exclude autoimmune paraneoplastic or non-paraneoplastic encephalitis (28-30) in the present case because the antibodies that are detected in these forms of encephalitis were not measured. However, hypereosinophilia has not been reported in association with these forms of encephalitis.

In conclusion, this case suggests that fatal encephalitis may develop in association with HES. Further study of similar cases is needed to elucidate the association between eosinophilia and encephalitis in HES.

The authors state that they have no Conflict of Interest (COI).

\section{Acknowledgement}

The authors thank Dr. Yoshitaka Uno for helpful comments, Ms. Hiromi Kondo and Ms. Chie Haga for technical assistance, and Mr. Yo Shoda and Ms. Kyoko Suzuki for photographic assistance. This work was supported by a grant-in-aid for scientific research from the Ministry of Education, Culture, Sports, Science and Technology (14570957) and a research grant from the Zikei Institute of Psychiatry.

\section{References}

1. Roufosse F. Hypereosinophilic syndrome variants: diagnostic and therapeutic considerations. Haematologica 94: 1188-1193, 2009.

2. Gleich GJ, Leiferman KM. The hypereosinophilic syndromes: current concepts and treatments. Br J Haematol 145: 271-285, 2009.

3. Lincoff NS, Schlesinger D. Recurrent optic neuritis as the presenting manifestation of primary hypereosinophilic syndrome: a report of two cases. J Neuroophthalmol 25: 116-121, 2005.

4. Tsai CP, Yeh HH, Tsai JJ, Lin KP, Wu ZA. Transverse myelitis and polyneuropathy in idiopathic hypereosinophilic syndrome. Muscle Nerve 16: 112-113, 1993.

5. Chou CW, Hsu SP, Chen MT, et al. Idiopathic hypereosinophilic syndrome with infiltration of cerebrospinal fluid by immature eosinophils: a case report and literature review. Surg Neurol $\mathbf{6 8}$ (Suppl 1): S52-S55, 2007.

6. Moore PM, Harley JB, Fauci AS. Neurologic dysfunction in the idiopathic hypereosinophilic syndrome. Ann Intern Med 102: 109114, 1985.

7. Kono Y, Itoh Y. Diffusion-weighted imaging of encephalopathy related to idiopathic hypereosinophilic syndrome. Clin Neurol Neurosurg 111: 551-553, 2009.

8. Kwon SU, Kim JC, Kim JS. Sequential magnetic resonance imaging findings in hypereosinophilia-induced encephalopathy. J Neurol 248: 279-284, 2001.

9. Weingarten JS, O'Sheal SF, Margolis WS. Eosinophilic meningitis and the hypereosinophilic syndrome. Case report and review of the literature. Am J Med 78: 674-676, 1985.

10. Kanamori M, Suzuki H, Sato I, Ohyama K, Tezuka F, Katakura R. A case of idiopathic hypereosinophilic syndrome with leptomeningeal dissemination and intraventricular mass lesion: an autopsy report. Clin Neuropathol 28: 197-202, 2009.

11. Kaplan PW, Waterbury L, Kawas C, Bolla-Wilson K, Durack D. Reversible dementia with idiopathic hypereosinophilic syndrome. Neurology 39: 1388-1391, 1989.

12. Kim HY, Kim YM, Kim SH, Kim HJ. Encephalitis in idiopathic hypereosinophilic syndrome in childhood. Pediatr Radiol 40 (Suppl 1): 130-133, 2010.

13. Tamaru $Y$, Nakashita $M$, Ito $H$, Okumura R, Matsumoto $S$, Imai $T$. Spontaneous remission of a massive CNS inflammation with eosinophilic infiltrate. Intern Med 42: 424-427, 2003.

14. Battineni ML, Galetta SL, Oh J, et al. Idiopathic hypereosinophilic syndrome with skull base involvement. AJNR Am J Neuroradiol 28: 971-973, 2007.

15. McMillan HJ, Johnston DL, Doja A. Watershed infarction due to acute hypereosinophilia. Neurology 70: 80-82, 2008.

16. Grigoryan M, Geisler SD, St Louis EK, Baumbach GL, Davis PH. Cerebral arteriolar thromboembolism in idiopathic hypereosinophilic syndrome. Arch Neurol 66: 528-531, 2009.

17. Sarazin M, Caumes E, Cohen A, Amarenco P. Multiple microembolic borderzone brain infarctions and endomyocardial fibrosis in idiopathic hypereosinophilic syndrome and in Schistosoma mansoni infestation. J Neurol Neurosurg Psychiatry 75: 305-307, 2004.

18. Takeuchi S, Takasato $\mathrm{Y}$, Masaoka $\mathrm{H}$, et al. Middle cerebral artery occlusion resulting from hypereosinophilic syndrome. J Clin Neurosci 17: 377-378, 2010.

19. Lee EJ, Lee YJ, Lee SR, Park DW, Kim HY. Hypereosinophilia with multiple thromboembolic cerebral infarcts and focal intracerebral hemorrhage. Korean J Radiol 10: 511-514, 2009.

20. Schulman H, Hertzog L, Zirkin H, Hertzanu Y. Cerebral sinovenous thrombosis in the idiopathic hypereosinophilic syndrome in childhood. Pediatr Radiol 29: 595-597, 1999.

21. Sakuta R, Tomita Y, Ohashi M, Nagai T, Murakami N. Idiopathic hypereosinophilic syndrome complicated by central sinovenous thrombosis. Brain Dev 29: 182-184, 2007.

22. Yoshikawa H. Neuropathological findings in hypereosinophilic syndrome. Intern Med 42: 381-382, 2003.

23. Fujino $Y$, Nakajima $M$, Inoue $H$, Kusuhara $T$, Yamada T. Human herpesvirus 6 encephalitis associated with hypersensitivity syndrome. Ann Neurol 51: 771-774, 2002.

24. Masaki T, Fukunaga A, Tohyama M, et al. Human herpes virus 6 encephalitis in allopurinol-induced hypersensitivity syndrome. Acta Derm Venereol 83: 128-131, 2003.

25. Ito T, Ooishi C, Chiba A, Sakuta M, Sakuma K, Shiohara T. Limbic encephalitis associated with drug-induced hypersensitivity syndrome due to phenobarbital--a case report. Rinshou Shinkeigaku (Clin Neurol) 45: 495-501, 2005 (in Japanese, Abstract in English). 
26. Sakuma K, Kano Y, Fukuhara M, Shiohara T. Syndrome of inappropriate secretion of antidiuretic hormone associated with limbic encephalitis in a patient with drug-induced hypersensitivity syndrome. Clin Exp Dermatol 33: 287-290, 2008.

27. Descamps V, Collot S, Houhou N, Ranger-Rogez S. Human herpesvirus-6 encephalitis associated with hypersensitivity syndrome. Ann Neurol 53: 280, 2003.

28. Dalmau J, Graus F, Villarejo A, et al. Clinical analysis of anti-Ma 2-associated encephalitis. Brain 127: 1831-1844, 2004.
29. Irani SR, Bera $K$, Waters $P$, et al. N-methyl-D-aspartate antibody encephalitis: temporal progression of clinical and paraclinical observations in a predominantly non-paraneoplastic disorder of both sexes. Brain 133: 1655-1667, 2010.

30. Irani SR, Alexander S, Waters $P$, et al. Antibodies to Kv1 potassium channel-complex proteins leucine-rich, glioma inactivated 1 protein and contactin-associated protein-2 in limbic encephalitis, Morvan's syndrome and acquired neuromyotonia. Brain 133: 2734-2748, 2010.

(C) 2011 The Japanese Society of Internal Medicine http://www.naika.or.jp/imindex.html 